\title{
ON THE SOLUTION OF A CLASS OF SECOND-ORDER QUASI-LINEAR PDES AND THE GAUSS EQUATION
}

\author{
A. R. SELVARATNAM ${ }^{1}, M$. VLIEG-HULSTMAN ${ }^{1}$, B. VAN-BRUNT ${ }^{1}$ \\ and W. D. HALFORD ${ }^{1}$
}

(Received 17 March 1997; revised 14 January 2000)

\begin{abstract}
Gauss' Theorema Egregium produces a partial differential equation which relates the Gaussian curvature $K$ to components of the metric tensor and its derivatives. Well-known partial differential equations (PDEs) such as the Schrödinger equation and the sine-Gordon equation can be derived from Gauss' equation for specific choices of $\boldsymbol{K}$ and coördinate systems. In this paper we consider a class of Bäcklund Transformations which corresponds to coördinate transformations on surfaces with a given Gaussian curvature. These Bäcklund Transformations lead to the construction of solutions to certain classes of non-linear second order PDEs of hyperbolic type by identifying these PDEs as the Gauss equation in some coördinate system. The possibility of solving the Cauchy Problem has also been explored for these classes of equations.
\end{abstract}

\section{Introduction}

In this paper we develop a method for solving certain types of PDEs based on finding Bäcklund Transformations [10]. The PDE from Gauss' Theorema Egregium [1], the Gauss equation, plays a central rôle in this method. The basic idea is that, if a PDE can be interpreted as the Gauss equation in a particular coördinate system for a surface of known Gaussian curvature, then a solution may be obtainable by the use of Bäcklund Transformations which, in essence, can be interpreted as coördinate transformations on surfaces having the same Gaussian curvature.

Many nonlinear and some linear PDEs of interest correspond to the Gauss equation. Konopelchenko [6] showed that the Schrödinger equation

$$
\psi_{x x}+K \psi=0
$$

\footnotetext{
'Department of Mathematics, Massey University, Paimerston North, New Zealand.

(C) Australian Mathematical Society 2001, Serial-fee code 0334-2700/01
} 
where $\psi$ and $K$ are both functions of $x$ and $y$, corresponds to the Gauss equation for surfaces of Gaussian curvature $K$ in geodesic coördinates. The sine-Gordon equation corresponds to Gauss' equation for a pseudosphere in a Tchebychef net [11, 12] coördinate system.

Given a nonlinear PDE, it may be possible to interpret it as a statement of Gauss' Theorema Egregium for some $K$, which is known in terms of the given coördinates and possibly the unknown function. If this PDE is the Gauss equation in disguise, then a coördinate transformation to geodesic coördinates yields the Schrödinger equation (which may be easier to solve than the given PDE).

We use this technique to solve a class of second-order quasi-linear partial differential equations of the hyperbolic type. As an example, we obtain a family of solutions for the sine-Gordon equation.

Finally, solutions to the Cauchy problem are discussed and the sine-Gordon equation is used as an example.

\section{Solving a class of second-order quasi-linear PDEs}

The Gauss equation, in terms of $E, F$ and $G$, the coefficients of the first fundamental form on a surface with local coördinates $u$ and $v$, is

$$
K(u, v)=\frac{1}{2 H}\left\{\left(\frac{F}{H E} E_{v}-\frac{1}{H} G_{u}\right)_{u}+\left(\frac{2}{H} F_{u}-\frac{1}{H} E_{v}-\frac{F}{H E} E_{u}\right)_{v}\right\},
$$

where $K$ is the Gaussian curvature and $H^{2}=E G-F^{2}$. Suppose $E, F$ and $G$ are of the form $E(\phi), F(\phi)$ and $G(\phi)$. Here $\phi$ is some function of $u$ and $v$. Under this assumption (1) becomes

$$
G_{\phi} \phi_{u u}-2 F_{\phi} \phi_{u v}+E_{\phi} \phi_{v v}+2 K H^{2}+\Theta\left(u, v, \phi, \phi_{u}, \phi_{v}\right)=0,
$$

where

$$
\Theta\left(u, v, \phi, \phi_{u}, \phi_{v}\right)=\Theta_{1}(u, v, \phi)\left(\phi_{u}\right)^{2}+\Theta_{2}(u, v, \phi)\left(\phi_{v}\right)^{2}+\Theta_{3}(u, v, \phi) \phi_{u} \phi_{v}
$$

and

$$
\begin{aligned}
& \Theta_{1}(u, v, \phi)=-\frac{\left\{G_{\phi} E_{\phi} G+E\left(G_{\phi}\right)^{2}-2 G_{\phi} F_{\phi} F-2 H^{2} G_{\phi \phi}\right\}}{2 H^{2}}, \\
& \Theta_{2}(u, v, \phi)=-\frac{\left\{G_{\phi} E_{\phi} E+G\left(E_{\phi}\right)^{2}-2 E_{\phi} F_{\phi} F-2 H^{2} E_{\phi \phi}\right\}}{2 H^{2}}, \\
& \Theta_{3}(u, v, \phi)=\frac{\left\{F_{\phi} E_{\phi} G+F_{\phi} G_{\phi} E-2 F\left(F_{\phi}\right)^{2}-2 H^{2} F_{\phi \phi}\right\}}{H^{2}} .
\end{aligned}
$$


Equation (2) is of the form

$$
A \phi_{u u}+2 B \phi_{u v}+C \phi_{v v}+D=0,
$$

which is the general form for a second-order quasi-linear PDE with coefficients $A, B$ and $C$ depending on $u, v$ and $\phi$ and $D$ depending on $u, v, \phi, \phi_{u}$ and $\phi_{v}$.

If $B^{2}-A C$ does not change sign, (3) can be classified into one of three types: hyperbolic, parabolic or elliptic, corresponding to $B^{2}-A C>0, B^{2}-A C=0$, or $B^{2}-A C<0$ respectively. We shall focus on the hyperbolic case.

\section{Equations of hyperbolic type}

Consider a second-order quasi-linear hyperbolic PDE of the type

$$
\phi_{u v}=f\left(\phi, \phi_{u}, \phi_{v}\right) \text {. }
$$

Equation (2) will be of this form if $F_{\phi} \neq 0$ and either

(a) $E_{\phi}=G_{\phi}=0$ or

(b) $G_{\phi} \phi_{u u}+E_{\phi} \phi_{v v}=0$.

In this paper we shall consider only the first case and find the conditions under which (4) can be solved by our method. In this case $E=$ constant and $G=$ constant.

By an appropriate scaling of the coördinates $u$ and $v$ we can put $E$ and $G$ equal and without loss of generality we thus take $E=1$ and $G=1$. This choice of $E$ and $G$ corresponds to a Tchebychef net coördinate system and (2) reduces to

$$
\phi_{u v}=\frac{K H^{2}}{F_{\phi}}-\frac{1}{F_{\phi}}\left\{F_{\phi \phi}+\frac{F\left(F_{\phi}\right)^{2}}{H^{2}}\right\} \phi_{u} \phi_{v} .
$$

Equation (5) is of the form

$$
\phi_{u v}=M(\phi)+A(\phi) \phi_{u} \phi_{v},
$$

where

$$
M(\phi)=\frac{K\left(1-F^{2}\right)}{F_{\phi}}
$$

and

$$
A(\phi)=\frac{-1}{F_{\phi}}\left\{F_{\phi \phi}+\frac{F\left(F_{\phi}\right)^{2}}{\left(1-F^{2}\right)}\right\} .
$$

The above arguments indicate that a given second-order quasi-linear hyperbolic PDE of the form (6) can be identified as the Gauss equation, where the $(u, v)$ coördinate 
system corresponds to a Tchebychef net $(E=1, G=1$ and $F=F(\phi))$, on a surface of curvature

$$
K=\frac{M(\phi) F_{\phi}}{1-F^{2}}
$$

where the function $F$ can be determined from (8).

The angle $\chi$ between the coördinate lines on a surface described by $\mathbf{r}(u, v)$ is given by

where $\chi$ depends on $\phi$; thus

$$
\frac{\mathbf{r}_{u} \cdot \mathbf{r}_{v}}{\left\|\mathbf{r}_{u}\right\|\left\|\mathbf{r}_{v}\right\|}=\cos \chi
$$

$$
F=\cos \chi(\phi)
$$

Substituting (9) into (8) and solving for $\chi$ yields

$$
\chi(\phi)=\lambda \int \exp \left\{-\int_{0}^{\phi} A(\zeta) d \zeta\right\} d \phi+\lambda_{1}
$$

where $\lambda$ and $\lambda_{1}$ are arbitrary constants. We can thus identify the PDE given by (6) with a surface defined intrinsically by the quantities

$$
E=1, \quad F=\cos \chi(\phi), \quad G=1 \quad \text { and } \quad K=-\frac{M(\phi)}{\sin \chi(\phi)} \chi^{\prime}(\phi) .
$$

Note that if $A(\phi)=0$, then

$$
F=\cos \left(\lambda \phi+\lambda_{1}\right)
$$

From (6) and (10) we obtain

$$
\chi_{u v}=M_{1}(\chi)
$$

where

$$
M_{1}(\chi)=\lambda \exp \left\{-\int_{0}^{\phi} A(\zeta) d \zeta\right\} M(\phi)
$$

We can thus reduce a PDE of the form (6) to an equation of the form (13) by using a transformation defined by (10). It is therefore sufficient to investigate the case when $A(\phi)=0$. For this case (6) reduces to

$$
\phi_{u v}=M(\phi)
$$

and from (10) and (11) we have

$$
E=1, \quad F=\cos \left(\lambda \phi+\lambda_{1}\right), \quad G=1 \quad \text { and } \quad K=-\frac{\lambda M(\phi)}{\sin \left(\lambda \phi+\lambda_{1}\right)}
$$


The constants $\lambda$ and $\lambda_{1}$ correspond to the magnification and the shift of the angle between the characteristics respectively. If we choose $\lambda=1$ and $\lambda_{1}=0$ then we can identify a PDE of the form (14) as Gauss' equation on a surface $\Sigma$ with

$$
E=1, \quad F=\cos \phi, \quad G=1 \text { and } \quad K=-\frac{M(\phi)}{\sin \phi} .
$$

Next we derive a transformation on $\Sigma$ from a geodesic coördinate system $(x, y)$ to the $(u, v)$ coördinate system (Tchebychef net). Let $\hat{E}, \hat{F}$ and $\hat{G}$ be the coefficients of the first fundamental form in the geodesic coördinate system; thus, $\hat{E}=1$ and $\hat{F}=0$. Moreover, the Gauss equation reduces to the Schrödinger equation

$$
\hat{H}_{x x}+K(x, y) \hat{H}=0
$$

where $\hat{H}^{2}=\hat{E} \hat{G}-\hat{F}^{2}=\hat{G}$.

A solution to (14) can be obtained provided:

(i) a coördinate transformation between geodesic coördinates and Tchebychef net coördinates can be determined;

(ii) the Schrödinger equation can be solved.

The metric tensor transformation properties yield the following relations between the coördinates:

$$
\begin{gathered}
x_{u}^{2}+\hat{H}^{2} y_{u}^{2}=1, \\
x_{u} x_{v}+\hat{H}^{2} y_{u} y_{v}=F=\cos \phi, \\
x_{v}^{2}+\hat{H}^{2} y_{v}^{2}=1 .
\end{gathered}
$$

One solution to the above system of equations can be determined under the assumption that

$$
x_{u}=x_{v}=\left\{1-\hat{H}^{2}(x, y)\right\}^{1 / 2} \text { and } y_{u}^{2}=y_{v}^{2}=1,
$$

provided that $y_{u} \neq y_{v}$. Using (21) we can define a transformation implicitly by

$$
x=\int\left\{1-\hat{H}^{2}(x, y)\right\}^{1 / 2} d u+f(v)
$$

and

$$
y=u-v,
$$

where $f(v)$ satisfies

$$
f^{\prime}(v)+\frac{\partial}{\partial v}\left\{\int\left(1-\hat{H}^{2}(x, y)\right)^{1 / 2} d u\right\}=\left\{1-\hat{H}^{2}(x, y)\right\}^{1 / 2} .
$$


Clearly this transformation is non-singular since the Jacobian is non-zero. We also note that $\hat{H}<1$ unless $K=0$, that is, $M(\phi)=0$.

Equations (19) and (21) imply that

$$
\hat{H}=\sin \left(\frac{\phi}{2}\right)
$$

Substituting (24) into the Schrödinger equation (17) yields

$$
\phi_{x x}-\frac{1}{2} \tan \frac{\phi}{2}\left(\phi_{x}\right)^{2}=M(\phi) \sec ^{2} \frac{\phi}{2}
$$

The above equation is essentially a second-order ordinary differential equation which can be reduced to a first-order equation (using a Prüfer substitution) which in turn can be solved. Equation (25) thus yields the relation

$$
x=\frac{1}{2} \int \frac{\cos (\phi / 2)}{\left\{\int M(\phi) d \phi+c_{1}(y)\right\}^{1 / 2}} d \phi+c_{2}(y),
$$

where $c_{1}(y)$ and $c_{2}(y)$ are arbitrary functions of $y$.

Now (26) defines an implicit relationship

$$
\Lambda(x, y, \phi)=0
$$

between $x, y$ and $\phi$. Under the assumption that one set of values $x_{0}, y_{0}, \phi_{0}$ can be found to satisfy (27) and that, near $\left(x_{0}, y_{0}, \phi_{0}\right), \Lambda$ and its first partial derivatives are continuous and $\partial \Lambda / \partial \phi \neq 0$, the implicit function theorem [8] implies that in a region of the $x y$ plane containing $\left(x_{0}, y_{0}\right)$, there is precisely one differentiable function

$$
\phi=\alpha(x, y)
$$

which reduces (27) to an identity and is such that $\phi_{0}=\alpha\left(x_{0}, y_{0}\right)$. Under these assumptions (21) gives, using (23), (24) and (28),

$$
x_{u}=x_{v}=\cos (\alpha(x, u-v)) \text {, }
$$

where, by (26), $\alpha(x, y)$ contains the two arbitrary functions $c_{1}(y)$ and $c_{2}(y)$. Hence we have determined a coördinate transformation

$$
\begin{aligned}
& x=\beta(u, v), \\
& y=u-v .
\end{aligned}
$$

Note that the Jacobian $\partial(x, y) / \partial(u, v) \neq 0$, so that this transformation is invertible. The general solution to the given PDE (6) is

$$
\phi=\alpha(x, y)=\alpha(\beta(u, v), u-v)=\Phi(u, v) .
$$


Example: The sine-Gordon equation The sine-Gordon equation

$$
\phi_{u v}=\sin \phi,
$$

is of the form (14) with $M(\phi)=\sin \phi$ and $K=-1$. For this choice of $M(26)$ becomes

$$
x=\frac{1}{2} \int \frac{\cos (\phi / 2)}{\left.1-\cos \phi+c_{1}(y)\right\}^{1 / 2}} d \phi+c_{2}(y),
$$

where $c_{1}(y)$ and $c_{2}(y)$ are arbitrary functions of $y$.

Integrating (33) and solving for $\phi(x, y)$ yields

$$
\phi(x, y)=2 \sin ^{-1}\left\{\sqrt{\left(c_{1}(y)-1\right) / 2} \sinh \left\{x-c_{2}(y)\right\}\right\}
$$

hence (24) implies that

$$
\hat{H}=\left\{\sqrt{\left(c_{1}(y)-1\right) / 2} \sinh \left[x-c_{2}(y)\right\},\right.
$$

which is a solution to the Schrödinger equation $\hat{H}_{x x}-\hat{H}=0$. Equations (21) and (35) imply that

$$
x_{u}=x_{v}=\left\{1-\left(\left(c_{1}(y)-1\right) / 2\right) \sinh ^{2}\left(x-c_{2}(y)\right)\right\}^{1 / 2} .
$$

From (36) it follows that

$$
u+\rho(v)=(k / 2) F(\alpha, k)=(k / 2) \mathrm{sn}^{-1}(\sin \alpha, k),
$$

where $\rho(v)$ depends upon the arbitrary functions $c_{1}$ and $c_{2}$,

$$
\begin{gathered}
\rho^{\prime}(v)=\frac{\partial}{\partial v}\left\{\frac{k}{2} \operatorname{sn}^{-1}(\sin \alpha, k)\right\}, \\
\alpha=\sin ^{-1}\left\{(1 / k) \tanh \left(x-c_{2}(y)\right)\right\}
\end{gathered}
$$

and

$$
k=\sqrt{2 /\left(c_{1}(y)+1\right)} .
$$

Here, $F$ denotes an elliptic function of the first kind $[3,7]$. Equations (37) and (38) indicate that

$$
x=\tanh ^{-1}\{k \operatorname{sn}((2 / k)(u+\rho(v)), k)\}+c_{2}(y),
$$

where $y=u-v$. Using (34), (39) and (40) (after some algebraic manipulations) we thus obtain the family of solutions to the sine-Gordon equation:

$$
\phi(u, v)=2 \sin ^{-1}\left\{\frac{\operatorname{sn}((2 / k)(u+\rho(v)), k)}{\left\{1+\left(k^{2} /\left(1-k^{2}\right)\right) \mathrm{cn}^{2}((2 / k)(u+\rho(v)), k)\right\}^{1 / 2}}\right\},
$$

where $k$ is an arbitrary function of $u-v$ and $\rho(v)$ is an arbitrary function of $v$. 


\section{The Cauchy Problem for the sine-Gordon equation}

The Cauchy Problem [5] for (6) consists of solving this PDE for given initial data along a non-characteristic curve. Let $\Omega$ be a smooth non-intersecting curve defined in the plane in parametric form by $u=U(t)$ and $v=V(t)$. Let

$$
\phi_{u}=p \text { and } \phi_{v}=q
$$

and suppose that, on $\Omega$, the Cauchy data are

$$
\phi=\Phi(t), \quad p=P(t) \quad \text { and } \quad q=Q(t) .
$$

For compatibility it is required that

$$
\dot{\Phi}(t)=P(t) \dot{U}(t)+Q(t) \dot{V}(t),
$$

where - denotes differentiation with respect to $t$. The following system of equations must therefore be satisfied along $\Omega$ :

and

$$
\begin{aligned}
& \phi_{u v}=f(\Phi(t), P(t), Q(t)), \\
& \dot{U}(t) \phi_{u u}+\dot{V}(t) \phi_{v v}=\dot{P}(t),
\end{aligned}
$$

$$
\dot{U}(t) \phi_{u v}+\dot{V}(t) \phi_{v v}=\dot{Q}(t) .
$$

The Cauchy data are non-characteristic if

$$
\dot{U} \neq 0 \text { and } \quad \dot{V} \neq 0 .
$$

To solve the Cauchy problem for the sine-Gordon equation (32), we shall take $\phi$ as in (34) (using $(x, y)$ geodesic coördinates) rather than as in (41) which uses the Tchebychef net $(u, v)$, as the calculations for $\phi_{u}$ and $\phi_{v}$ are easier this way. Equation (34) implies that

$$
\phi_{u}+\phi_{v}=4 \sqrt{\left(c_{1}(y)-1\right) / 2} \cosh \left(x-c_{2}(y)\right)
$$

and

$$
\phi_{u}-\phi_{v}=\frac{\left\{-4\left(\left(c_{1}(y)-1\right) / 2\right) \cosh \left(x-c_{2}(y)\right) c_{2}^{\prime}(y)+\sinh \left(x-c_{2}(y)\right) c_{1}^{\prime}(y)\right\}}{2 \sqrt{\left(c_{1}(y)-1\right) / 2}\left\{1-\left(\left(c_{1}(y)-1\right) / 2\right) \sinh ^{2}\left(x-c_{2}(y)\right)\right\}^{1 / 2}} .
$$

Suppose now that the initial conditions in the $(u, v)$ coördinate system (Tchebychef net) $\phi_{u}=P(t)$ and $\phi_{v}=Q(t)$ are transformed to $P_{1}(t)$ and $Q_{1}(t)$ in the $(x, y)$ (geodesic) coördinate system. The last two expressions indicate that

$$
P_{1}+Q_{1}=4 \sqrt{\left(c_{1}\left(y_{0}\right)-1\right) / 2} \cosh \left(x_{0}-c_{2}\left(y_{0}\right)\right)
$$


and

$$
P_{1}-Q_{1}=\frac{\left\{-4\left(\left(c_{1}\left(y_{0}\right)-1\right) / 2\right) \cosh \left(x_{0}-c_{2}\left(y_{0}\right)\right) c_{2}^{\prime}\left(y_{0}\right)+\sinh \left(x_{0}-c_{2}\left(y_{0}\right)\right) c_{1}^{\prime}\left(y_{0}\right)\right\}}{\sqrt{\left(c_{1}\left(y_{0}\right)-1\right) / 2}\left\{1-\left(\left(c_{1}\left(y_{0}\right)-1\right) / 2\right) \sinh ^{2}\left(x_{0}-c_{2}\left(y_{0}\right)\right)\right\}^{1 / 2}}
$$

where $x_{0}(t)$ and $y_{0}(t)$ correspond to the initial curve in the $(x, y)$ geodesic coördinate system. Solving the above expression for $c_{1}^{\prime}\left(y_{0}\right)$ yields the first order differential equation

$$
c_{1}^{\prime}\left(y_{0}\right)=A\left\{1-\left(B-c_{1}\left(y_{0}\right)\right)^{2}\right\}^{1 / 2},
$$

where

$$
A=-\frac{\left(P_{1}-Q_{1}\right)}{2} \text { and } B=\left(\frac{P_{1}+Q_{1}}{2 \sqrt{2}}\right)^{2}
$$

In principle, the differential equation (45) can be solved for $c_{1}^{\prime}\left(y_{0}\right)$ in terms of $c_{2}\left(y_{0}\right)$. Equation (43) can then be used to determine $c_{2}\left(y_{0}\right)$. The Cauchy Problem for the sine-Gordon equation can thus be reduced to a problem involving the solution of a nonlinear first order ODE and inversions.

For a concrete example, suppose that $\Omega$ is parametrized in the Tchebychef net coördinate system by

$$
u+v=c_{0} \text { constant }
$$

and on $\Omega$,

$$
\phi=\Phi_{0}, \quad p=P_{0} \quad \text { and } q=Q_{0}
$$

where $\Phi_{0}, P_{0}$ and $Q_{0}$ are constants. It can be shown using (31) that the initial conditions given in (48), when transformed to the geodesic $(x, y)$ coördinates, are of the same form, that is,

$$
\phi=\Phi_{1}, \quad p=P_{1} \text { and } q=Q_{1}
$$

where $\Phi_{1}, P_{1}$ and $Q_{1}$ are constants. Now the quantities $A$ and $B$ (given in (46)) are also constants and (45) thus has the solution

$$
c_{1}\left(y_{0}\right)=B-\sin \left(A y_{0}+C\right)
$$

where the constant of integration is given by

$$
C=\sin ^{-1}\left\{2 \sin ^{2}\left(\Phi_{1} / 2\right)-1\right\}-A y_{0} .
$$


Generally,

$$
c_{1}(y)=B-\sin (A y+C)
$$

and, by (43),

$$
c_{2}(y)=x_{0}-\cosh ^{-1} \sqrt{\frac{B}{(B-1)-\sin (A y+C)}} .
$$

Hence a solution for the Cauchy Problem of the sine-Gordon equation in geodesic coördinates is given by (34), where $c_{1}(y)$ and $c_{2}(y)$ are defined by (50) and (51) respectively. To get a solution in terms of the original coördinates we can use (23) and (26) to express $x$ and $y$ in terms of $u$ and $v$.

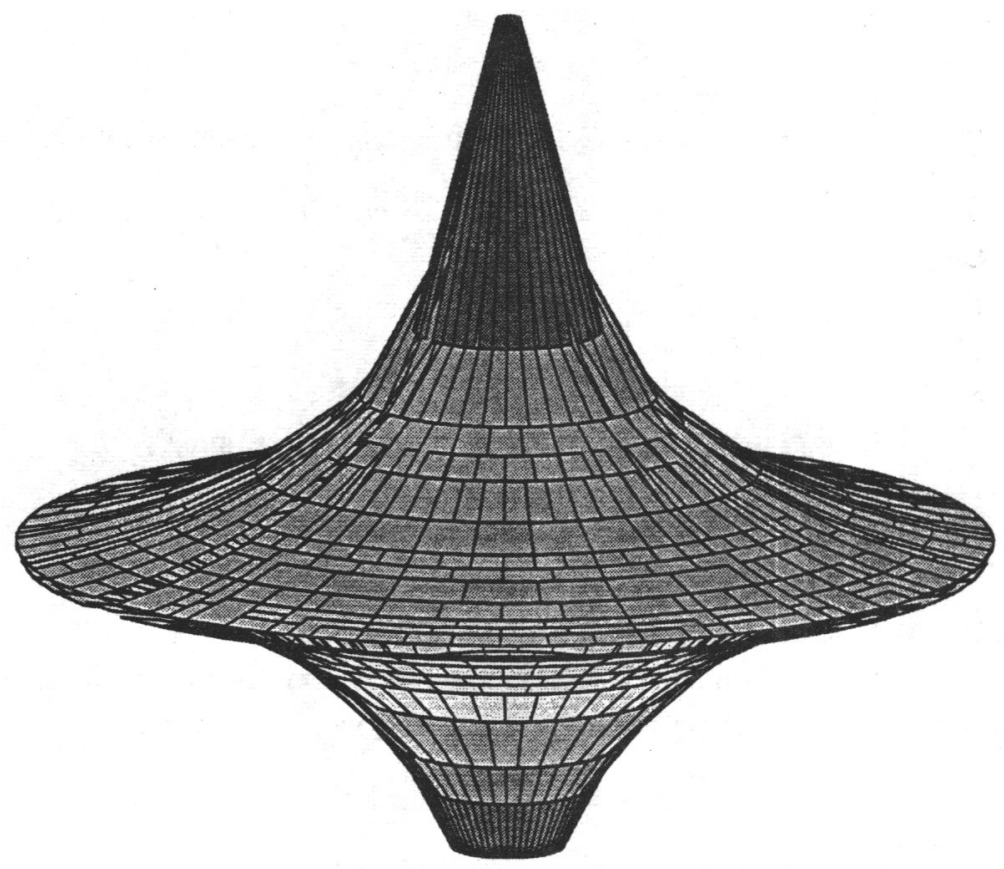

FIGURE 1. Beltrami surface in the $(X, Y)$ coördinate system

Though perhaps not obvious, this solution corresponds to a Beltrami surface [9] (see Figure 1). Recall that the Beltrami surface is a surface of revolution described by

$$
\mathbf{r}(X, Y)=(\sin X \cos Y, \sin X \sin Y, \cos X+\ln \tan (X / 2)),
$$

where the $(X, Y)$ coördinates correspond to the lines of curvature.

The coefficients $E, F$ and $G$ of the first fundamental form and the Gaussian curvature $K$ in this coördinate system are

$$
E=\cot ^{2} X, \quad F=0, \quad G=\sin ^{2} X \quad \text { and } \quad K=-1,
$$


and thus $H=\sqrt{E G-F^{2}}=\cos X$.

A transformation between the $(X, Y)$ coördinate system and the Tchebychef net $(u, v)$ coördinate system is defined by the system

$$
\begin{gathered}
X_{u}^{2} \cot ^{2} X+Y_{u}^{2} \sin ^{2} X=1, \\
X_{u} X_{v} \cot ^{2} X+Y_{u} Y_{v} \sin ^{2} X=\hat{F}=\cos \phi, \\
X_{v}^{2} \cot ^{2} X+Y_{v}^{2} \sin ^{2} X=1,
\end{gathered}
$$

and a solution to this system can be determined under the assumption that

$$
Y_{u}^{2}=Y_{v}^{2}=1
$$

Choosing

$$
Y=u-v
$$

(52) and (54) give $X_{u}=X_{v}=\sin X$. A suitable transformation is defined by (55) and

$$
u+v=\ln |\csc X-\cot X| \text {. }
$$

Equation (53) implies

$$
\phi=2 X
$$

further,

$$
\phi_{u}=\phi_{X} X_{u}+\phi_{Y} Y_{u}=2 \sin X, \quad \phi_{v}=2 \sin X
$$

and consequently

$$
\phi_{u v}=2 \sin X \cos X=\sin 2 X=\sin \phi,
$$

which is the sine-Gordon equation.

Any curve corresponding to $X=$ constant is a non-characteristic curve on the pseudosphere. Using the transformation equation (56), we have that

$$
u+v=c_{0} \quad \text { (constant). }
$$

This non-characteristic curve in $(u, v)$ coördinates (Tchebychef net) can be taken as the initial curve for the Cauchy Problem of the sine-Gordon equation. Also using (57) and (58) we see that

$$
\phi=c_{1}, \quad \phi_{u}=c_{2} \text { and } \phi_{v}=c_{3},
$$

where $c_{1}, c_{2}$ and $c_{3}$ are constants.

The last two equations are the same initial conditions as in (47) and (48). Since the Cauchy data are analytic the Cauchy-Kowalewski theorem [2] guarantees a unique solution. Thus the solution which we had for the Cauchy problem of the sine-Gordon equation corresponds to the Beltrami surface; the surface representation of this Cauchy Problem is now evident. 


\section{Conclusions}

The purpose of this paper is to use classical differential geometry in order to find Bäcklund Transformations and hence solve certain classes of non-linear partial differential equations. If a PDE can be interpreted as a statement of Gauss' theorem for some known coördinate system, then it may be possible to use some coördinate transformations to convert the PDE into one which is easier to solve. In this paper we have focused on a simple, specific case and used the sine-Gordon equation to illustrate the ideas. It is clear, however, that more complicated nonlinear PDEs can be attacked in this manner and that initial value problems can be reduced to problems involving inversions, quadratures and the solution of a first-order ODE.

\section{References}

[1] L. P. Eisenhart, A Treatise on the Differential Geometry of Curves and Surfaces (Ginn, 1909) (reprint 1960).

[2] P. R. Garabedian, Partial Differential Equations (John Wiley and Sons, 1964).

[3] I. S. Gradshteyn and I. M. Ryzhik, Table of Integrals, Series and Products (Academic Press, 1980).

[4] M. Johnston, "Geometry and the sine-Gordon equation", Technical Report AMR 95/16, University of New South Wales, Australia, 1995.

[5] J. Kevorkian, Partial Differential Equations, Analytical Solution Techniques (Chapman and Hall, 1993).

[6] B. G. Konopelchenko, "Solitons of curvature", Acta Applicandae Mathematicae 39 (1995) 379387.

[7] D. F. Lawden, Elliptic Functions and Applications (Springer-Verlag, 1989).

[8] L. Marden, Calculus of Several Variables (George Allen and Unwin, 1971).

[9] A. V. Pogorelov, Differential Geometry (P. Noordhoff, Groningen, The Netherlands, 1959).

[10] C. Rogers and W. F. Shadwick, Bäcklund Transformations and Their Applications (Academic Press, 1982).

[11] J. J. Stoker, Differential Geometry (John Wiley and Sons, 1989).

[12] D. J. Struik, Lectures on Classical Differential Geometry (Addison-Wesley, 1961). 